\title{
VISUAL LIGHT FROM THE ECLIPSING SUPERSOFT X-RAY SOURCE CAL 87
}

\author{
S. SCHANDL, E. MEYER-HOFMEISTER, F. MEYER \\ Max-Planck Institut für Astrophysik, Karl Schwarzschildstr. 1, \\ 85748 Garching, Germany
}

We calculate the visual light curve of CAL 87 based on the assumption that an accreting, steadily burning white dwarf irradiates the accretion disk and the secondary star, as suggested by van den Heuvel et al. (1992). The shape of the eclipse light curve and the absolute luminosities put strong constraints on the contributions of the various elements in the system. We find a reasonable fit is obtained with the following sources of visual light: a Roche lobe filling secondary star where the energy from irradiation is spread out over the surface, and an accretion disk with an optically thick, cold, clumpy spray which is caused by the massive accretion stream impinging on the disk (hot spot). This spray moving around the disk and illuminated by the white dwarf is found to be the brightest source of visual light in the binary and can account for the asymmetry in the light curve.

We calculate the emission of all contributing surfaces by assuming a blackbody radiation spectrum. For comparison with the observations we folded it with a visual filter function. At parts facing the white dwarf the irradiated flux is added to the viscous dissipation of the disk, the constant flux of the spray and the original stellar flux of the secondary. Energy transport on the stellar surface is approximated by spreading the irradiated flux weighted by a Gaussian kernel. For more details see Schandl, MeyerHofmeister \& Meyer (1996).

The simulation yields an inclination of about $77^{\circ}$ and supports the assumption that the white dwarf is permanently hidden by the disk.

\section{References}

Schandl S., Meyer-Hofmeister E., Meyer F., 1996, A\&A, submitted (and Green Report No. 912 of the Max-Planck Institut für Astrophysik, Garching, Germany)

Schmidtke, P.C., McGrath, T.K., Cowley, A.P., Frattare, L.M., 1993, PASP, 105, 863

van den Heuvel, E.P.J., Bhattacharya, D., Nomoto, K., Rappaport S.A., 1992, A\&A, 262, 97

A. Evans and J. H. Wood (eds.), Cataclysmic Variables and Related Objects, 425-426.

(c) 1996 Kluwer Academic Publishers. Printed in the Netherlands. 
TABLE 1. The System Parameters

\section{Facts:}

Orbital period

$$
\begin{aligned}
P & =10.6 \mathrm{~h} \\
d & =57.5 \mathrm{kpc}
\end{aligned}
$$

Distance

Assumed parameters (model of van den Heuvel et al. 1992):

Primary star (white dwarf)

Secondary star (Roche lobe filling main sequence star)

Mass accretion rate

Temperature of the non-irradiated Secondary star

$\Rightarrow$ disk size (0.8 Roche lobe)

$\Rightarrow$ luminosity of white dwarf

$$
\begin{aligned}
M_{1} & =0.75 \mathrm{M}_{\odot} \\
M_{2} & =1.5 \mathrm{M}_{\odot} \\
\dot{M} & =0.810^{-7} \mathrm{M}_{\odot} \mathrm{yr}^{-1} \\
T_{\star} & =8000 \mathrm{~K} \\
s & =5.610^{10} \mathrm{~cm} \\
L & =2.210^{37} \mathrm{erg} \mathrm{s}^{-1}
\end{aligned}
$$

Further parameters taken for the presented theoretical light curve:
Albedo (for star and disk)
$a=0.5$
Inclination
$i=77^{\circ}$



Figure 1. The visual light curve of CAL 87 . At primary minimum the observer sees the dark side of the secondary and a non-eclipsed part of the disk. At secondary minimum one sees the partial occultation of the illuminated secondary by the disk. The dots show the composite $V$ light curve where the photometry was done between 1985 November and 1992 December (from Schmidtke et al. 1993). The fit (solid line) consists of a secondary with sideways energy transport (dashed line) and the illuminated accretion disk with an additional hot spot region (dotted line). 